\title{
Efflux-mediated resistance to a benzothiadiazol derivative effective against Burkholderia cenocepacia
}

\author{
Viola C. Scoffone1, Olga Ryabova², Vadim Makarov², Paolo ladarola1, Marco Fumagalli', \\ Marco Fondi ${ }^{3}$, Renato Fani ${ }^{3}$, Edda De Rossi ${ }^{1}$, Giovanna Riccardi ${ }^{1}$ and Silvia Buroni ${ }^{*}$ * \\ 'Laboratory of Molecular Microbiology, Dipartimento di Biologia e Biotecnologie "Lazzaro Spallanzani," Università degli Studi \\ di Pavia, Pavia, Italy, ${ }^{2}$ Bakh Institute of Biochemistry, Russian Academy of Science, Moscow, Russia, ${ }^{3}$ Department of \\ Biology, University of Florence, Florence, Italy
}

\section{OPEN ACCESS}

Edited by:

Paolo Visca,

Roma Tre University, Italy

Reviewed by:

Pierre Cornelis,

Vrije Universiteit Brussel, Belgium Giordano Rampioni,

Roma Tre University, Italy

${ }^{*}$ Correspondence: Silvia Buroni,

Laboratory of Molecular Microbiology, Dipartimento di Biologia e Biotecnologie "Lazzaro Spallanzani,"

Università degli Studi di Pavia, Via Ferrata, 1-27100 Pavia, Italy silvia.buroni@unipv.it

Specialty section: This article was submitted to Antimicrobials, Resistance and Chemotherapy,

a section of the journal Frontiers in Microbiology

Received: 08 May 2015 Accepted: 24 July 2015 Published: 05 August 2015

Citation:

Scoffone VC, Ryabova O, Makarov V ladarola P, Fumagalli M, Fondi $M$, Fani R, De Rossi E, Riccardi $G$ and Buroni S (2015) Efflux-mediated resistance to a benzothiadiazol derivative effective against Burkholderia cenocepacia. Front. Microbiol. 6:815 doi: 10.3389/fmicb.2015.00815
Burkholderia cenocepacia is a major concern for people suffering from cystic fibrosis as it contributes to serious respiratory tract infections. The lack of drugs effective against this opportunistic pathogen, along with the high level of resistance to multiple antibiotics, render the treatment of these infections particularly difficult. Here a new compound, belonging to the 2,1,3-benzothiadiazol-5-yl family (10126109), with a bactericidal effect and a minimal inhibitory concentration (MIC) of $8 \mu \mathrm{g} / \mathrm{ml}$ against B. cenocepacia, is described. The compound is not cytotoxic and effective against $B$. cenocepacia clinical isolates and members of all the known $B$. cepacia complex species. Spontaneous mutants resistant to 10126109 were isolated and mutations in the MerR transcriptional regulator BCAM1948 were identified. In this way, a mechanism of resistance to this new molecule was described, which relies on the overexpression of the RND-9 efflux pump. Indeed, rnd-9 overexpression was confirmed by quantitative reverse transcription PCR, and RND-9 was identified in the membrane fractions of the mutant strains. Moreover, the increase in the MIC values of different drugs in the mutant strains, together with complementation experiments, suggested the involvement of RND-9 in the efflux of 10126109 , thus indicating again the central role of efflux transporters in $B$. cenocepacia drug resistance.

Keywords: drug resistance, new antimicrobials, Gram-negatives, cystic fibrosis, efflux pumps, Burkholderia

\section{Introduction}

Burkholderia cenocepacia, together with B. multivorans, contributes to serious respiratory tract infections in people suffering from cystic fibrosis (CF; LiPuma, 2010). Worldwide about 80000 people are affected by CF, with a life expectancy of about 50 years; a proven effective therapy aimed at correcting the basic genetic defect is still lacking (Dodge et al., 2007). The disease causes the formation of thick and sticky mucus especially in the lungs, leading to serious infections that in 2009 in Europe accounted for the $45 \%$ of CF deaths ${ }^{1}$. In particular, B. cenocepacia is one of the worst CF-associated pathogens, as it carries the greatest risk of mortality, and it can lead to a fatal pneumonia called the "cepacia syndrome." Moreover, the low percentage of post-transplantation survival among B. cenocepacia infected patients makes the infection due to this bacterium a contraindication for transplantation in most hospitals (Noone, 2008;

\footnotetext{
${ }^{1}$ https://www.ecfs.eu/projects/ecfs-patient-registry/annual-reports
} 
De Soyza et al., 2010). Finally, due to its high level of resistance to most antibiotics (aminoglycosides, polymyxins, and $\beta$-lactams) it is particularly difficult to eradicate (Saiman et al., 2003). Accordingly, treatment of $B$. cenocepacia infections is often unsuccessful and triple antibiotic combination therapy is only aimed at decreasing bacterial load (Aaron et al., 2000).

Active transport is now recognized as a major cause of antimicrobial resistance in bacteria (Higgins, 2007). In $B$. cenocepacia the transporters belonging to the RND (Resistance-Nodulation-Cell Division) family are encoded by 16 operons (Perrin et al., 2013). We previously showed, by gene inactivation, the importance of RND-3 (BCAL167476) and RND-4 (BCAL2820-22) in the intrinsic resistance of B. cenocepacia J2315 as well as their involvement in the accumulation of quorum sensing molecules in the medium (Buroni et al., 2009). More recently, RND-4 has also been shown to be responsible for the resistance to a new antitubercular thiopyridine compound effective against B. cenocepacia (Scoffone et al., 2014). In another recent work, the effect of the deletion of the 16 RND operons was evaluated both in planktonic and sessile cells (Buroni et al., 2014). In particular, RND-3 and RND-4 have been shown to play a major role in the resistance to various antimicrobial drugs in planktonic $B$. cenocepacia J2315, while RND-3, RND-8, and RND-9 were needed to protect sessile cells against tobramycin or ciprofloxacin (Buroni et al., 2014).

In this study, a new molecule effective against $B$. cenocepacia J2315 is described. The compound belongs to the 2,1,3benzothiadiazol-5-yl family and was named 10126109. A mechanism of resistance to this new molecule is described, which relies on the overexpression of the RND-9 efflux pump, thus indicating, once again, the central role of efflux transporters in B. cenocepacia drug resistance.

\section{Materials and Methods}

\section{Bacterial Strains and Growth Conditions}

Burkholderia cenocepacia strains and B. cepacia complex (Bcc) species were grown in Luria-Bertani (LB) medium (Difco), with shaking at $200 \mathrm{rpm}$, or on LB agar, at $37^{\circ} \mathrm{C}$. B. cenocepacia J2315 was used as the wild-type (WT) strain.

\section{Synthesis of Methyl [(4-nitro-2,1,3- benzothiadiazol-5-yl)thio]acetate (10126109)}

Solution of $1.43 \mathrm{~g}$ ( $10 \mathrm{mmol})$ of 2-amino-4-chloroaniline in the mixture of $5.4 \mathrm{ml}$ thionyl chloride and $0.35 \mathrm{ml}$ of concentrated sulfuric acid was refluxed for $1 \mathrm{~h}$. The reaction mixture was cooled to $35^{\circ} \mathrm{C}$, mixed with $4.3 \mathrm{ml}$ of concentrated sulfuric acid and stored for $20 \mathrm{~min}$. This solution was treated by mixture of $1.43 \mathrm{ml}$ (32 mmol) fumigating nitric acid and $2.2 \mathrm{ml}$ concentrated sulfuric acid at $20-25^{\circ} \mathrm{C}$ for $10 \mathrm{~min}$. The reaction mixture was stored at $25-30^{\circ} \mathrm{C}$ for $30 \mathrm{~min}$ and poured to ice water. Gray precipitate was filtered off, washed by water and methanol and recrystallised from methanol. The yield of 5-chloro-4-nitro-2,1,3benzothiadiazole is $1.6 \mathrm{~g}(74 \%)$. mp: $145-147^{\circ} \mathrm{C}$.
Suspension of $0.5 \mathrm{~g}(2.32 \mathrm{mmol})$ of 5-chloro-4-nitro-2,1,3benzothiadiazole, $0.32 \mathrm{ml}(3.5 \mathrm{mmol})$ of methyl mercaptoacetate and $0.45 \mathrm{~g}(3.5 \mathrm{mmol})$ of potassium carbonate in $25 \mathrm{ml}$ of acetonitrile was stored for $30 \mathrm{~min}$ at room temperature. The reaction mixture was poured in $100 \mathrm{ml}$ of cold water and yellow precipitate was filtered off. The yield of methyl [(4-nitro-2,1,3benzothiadiazol-5-yl)thio] acetate is $0.17 \mathrm{~g}(24 \%) . \mathrm{mp}: 121-123^{\circ} \mathrm{C}$ (methanol); MS (m/z): $285\left(\mathrm{M}^{+}\right) ;{ }^{1} \mathrm{H}$ NMR (DMSO-d 6 ): $\delta \delta 8.22$ $(1 \mathrm{H}, \mathrm{d}, J=8.1 \mathrm{~Hz}, \mathrm{CH}), 7.67(1 \mathrm{H}, \mathrm{d}, J=8.8 \mathrm{~Hz}, \mathrm{CH}), 3.89(2 \mathrm{H}$, s, $\left.\mathrm{CH}_{2}\right), 3.71\left(3 \mathrm{H}, \mathrm{s}, \mathrm{CH}_{3}\right)$ ppm. Anal. $\mathrm{C}_{9} \mathrm{H}_{7} \mathrm{~N}_{3} \mathrm{O}_{4} \mathrm{~S}_{2}, \mathrm{C}, \mathrm{H}, \mathrm{N}$.

\section{Determination of Cytotoxicity}

HeLa Ohio cells were seeded at $2.4 \times 10^{4}$ cells/well in 96well flat-bottomed microtiter plates. To determine the 50\% cytotoxic concentration $\left(\mathrm{CC}_{50}\right)$, 2-days-old confluent $\mathrm{HeLa}$ cell monolayer were incubated with serial dilutions (factor 2, each concentration in duplicate) of the 10126109 compound for $72 \mathrm{~h}\left(37^{\circ} \mathrm{C}, 5 \% \mathrm{CO}_{2}\right)$. Then, the cells were fixed and stained with a crystal violet formalin solution. Cytotoxicity was quantified spectrophotometrically with a plate reader as described previously (Schmidtke et al., 2001).

The potential mutagenicity was assessed using the SOSchromotest at $50 \mu \mathrm{M}$ as previously described (Quillardet et al., 1982).

\section{Minimal Inhibitory Concentrations (MIC) Determination}

The effectiveness of 10126109 compound against B. cenocepacia $\mathrm{J} 2315$, B. cenocepacia clinical isolates and Bcc species, was assessed determining MICs. The experiment was performed with the twofold microdilution method in U-bottom 96well microtiter plates, and inoculating about $10^{5} \mathrm{CFU}$ in LB medium, using concentrations ranging from 1 to $256 \mu \mathrm{g} / \mathrm{ml}$. The microtiter plates were incubated at $37^{\circ} \mathrm{C}$ for 2 days and growth was determined by the resazurin method (Martin et al., 2006). A solution of resazurin sodium salt (Sigma-Aldrich) was prepared at $0.01 \%$ in distilled water and filter-sterilized. $30 \mu \mathrm{L}$ of resazurin solution were added to each well after 2 days of incubation at $37^{\circ} \mathrm{C}$, and the microtiters were reincubated at the same temperature for about $4 \mathrm{~h}$. The MIC was defined as the lowest concentration of the drug that prevented a change in color from blue to pink, which indicates the growth of bacteria.

The same results were confirmed also by streaking $1 \times 10^{4}$ cells onto LB agar containing twofold dilutions of the drug.

To obtain the resistance profile of $B$. cenocepacia mutants, the following compounds, at concentrations ranging from 2 to $256 \mu \mathrm{g} / \mathrm{ml}$, were tested: chloramphenicol, ciprofloxacin, levofloxacin, nalidixic acid, norfloxacin, and sparfloxacin. All antibiotics were purchased from Sigma-Aldrich. In all experiments the results represent the average of three independent replicates.

\section{Time-Killing Curve}

Time-killing curve of B. cenocepacia J2315 toward 10126109 was performed by the broth macrodilution method, as described by Silva et al. (2011). Briefly, 0.5, 1, 2, and 4 multiples of the MIC 
( $8 \mu \mathrm{g} / \mathrm{ml}$ ) were used. $20 \mathrm{ml}$ of LB broth with the appropriate 10126109 concentrations were inoculated with exponentially grown $B$. cenocepacia cells, to yield a final concentration of approximately $1 \times 10^{7} \mathrm{CFU}$ per $\mathrm{ml}$. The cultures were incubated at $37^{\circ} \mathrm{C}$, and aliquots were removed at $0,2,4,6,8,24$, 28 , and $32 \mathrm{~h}$ for the determination of viable counts. Serial dilutions were spread on LB solid medium and the plates were incubated at $37^{\circ} \mathrm{C}$ for 2 days. Then the number of colonies was determined.

Killing curves were constructed by plotting the log10 CFU $\mathrm{ml}^{-1}$ vs. time. Bactericidal activity was defined as a reduction of $99.9 \%(\geq 3 \log 10)$ of the total number of $\mathrm{CFU} \mathrm{ml} \mathrm{m}^{-1}$ in the original inoculum (NCCLS, 1999). Bacteriostatic activity was defined as maintenance of the original inoculum concentration or a reduction of less than $99.9 \%(<3 \log 10)$ of the total number of CFU ml $\mathrm{m}^{-1}$ in the original inoculum.

\section{Isolation of $B$. cenocepacia Mutants Resistant to 10126109}

Burkholderia cenocepacia resistant mutants were isolated by plating about $10^{9} \mathrm{CFU}$ of WT culture on LB agar, containing different concentrations of 10126109 compound, ranging from 4 to 20 -fold the MIC. Plates were incubated at $37^{\circ} \mathrm{C}$ for at least 3 weeks. The resistance profile of each strain was confirmed by growing the colonies in liquid LB medium containing the same concentration of molecule at which the strains were isolated, and re-streaking the cells onto solid medium containing 10126109.

\section{Genomic DNA Extraction and Sequencing}

Genomic DNA was extracted from B. cenocepacia J2315 and spontaneous mutants using Bacteria DNA Preparation Kit (Jena Bioscience). $10^{9}$ cells were harvested by centrifugation and treated as described in the manufacturer's protocol. The DNA was resuspended in Tris-EDTA (TE) and used for sequencing purpose. Quality and concentration of each sample were assessed using spectrophotometer and agarose gel electrophoresis. All genomes were sequenced through Illumina Solexa technology (IGA Technology Service Srl). Reads quality was assessed using FastQC toolkit ${ }^{2}$. Reads trimming was performed using the dynamic trimming approach implemented in SolexQA (Cox et al., 2010), using a Phred score of 30 as the base call quality threshold. Trimmed reads were then mapped on the reference genome using Mosaik aligner with default parameters (Lee et al., 2014). Only those SNPs that (i) were not present in the WT strain, (ii) were supported by, at least, eight reads, and (iii) had a support greater than $70 \%$ were considered for further analysis. SNPs calling was performed using VarScan (Koboldt et al., 2009).

Mutations were confirmed by sequencing PCR amplicons using the following primer pairs with HotStar HiFidelity Polymerase kit (Qiagen): BCAM1948FOR (5'-ATGGGTATCC AGGAAACC-3') and BCAM1948REV (5'-GAGTCGCTGCAG TTCCTCGA-3') for SB5 and SB36 mutants; BCAM1948SB34F (5'-AAATCGCCGAGCAGCTCGTCAGC-3') and BCAM194 8SB34R (5'-AGCCGGACGACATCGCGGACGC-3') for SB34

${ }^{2}$ http://www.bioinformatics.babraham.ac.uk/projects/fastqc/ mutant; BCAM1948SB6F (5'-TAAACTGCCGCGTAGCAT-3') and BCAM1948REV for SB6 mutant.

\section{RNA Purification and Reverse Transcription (RT)}

For the Quantitative reverse transcription PCR (qRT-PCR) experiment, WT and mutant $B$. cenocepacia cells $\left(1 \times 10^{9} \mathrm{CFU}\right)$ were collected by centrifugation. Total RNA was extracted using the RiboPure Bacteria Kit (Ambion), following the manufacturer's instructions. A $30 \mathrm{~min}$ incubation of each sample with DNase I (Ambion) was performed, following the manufacturer's protocol. RNA quality and concentration were assessed using both agarose gel electrophoresis and the spectrophotometric determination. One-microgram of total RNA was used for cDNA generation using the QuantiTect reverse Transcription kit (Qiagen) according to the manufacturer's instructions, but diluting the cDNA 1:2 before using for qRT-PCR.

\section{Quantitative Reverse Transcription PCR (qRT-PCR)}

For each strain, qRT-PCR experiments on BCAM1946 gene were performed. The following primers were used: BCAM1946Rtfor (5'-TGCTCGTCGTGATCCTGTTT-3') and BCAM1946Rtrev (5'-CGAACAGCGTGAGCGTATTG-3'). All reactions were performed on a Rotor-Gene-6000 cycler (Corbett), using Quanti-Tect SYBR Green PCR Kit (Qiagen), according to manufacturer's instructions. Fifteen-microliter were used as a final volume for each reaction. Cycling conditions were: $95^{\circ} \mathrm{C}$ for $15 \mathrm{~min}$ ( 1 cycle), $94^{\circ} \mathrm{C}$ for $15 \mathrm{~s}$ followed by $54^{\circ} \mathrm{C}$ for $30 \mathrm{~s}$ and $72^{\circ} \mathrm{C}$ for $30 \mathrm{~s}$ (40 cycles). A melting curve analysis was included at the end of each run. Each sample was spotted in triplicate and a reference gene, as well as control samples without cDNA, were included in each experiment. BCAM0918 (rpoD) gene was used as reference gene with the following primers: 0918F (5'-GCCAACCTGCGTCTCGT-3') and 0918R (5'AACTTGTCCACCGCCTT-3'), using an annealing temperature of $50^{\circ} \mathrm{C}$. The fold difference in gene expression between the mutants and the WT was assessed using the comparative Ct-method (Livak and Schmittgen, 2001). The results are the average of three independent replicates. To determine if differences in expression were significant $(P<0.05)$ the Mann-Whitney test was used.

\section{Complementation}

For complementation experiments the entire BCAM1948 gene was PCR amplified from $B$. cenocepacia J2315 genomic DNA using primers 1948compF (5'-TTCATATGTAAACTG CCGCGTAGCAT-3') and 1948compR (5'-TTGGATCCCGT CATTCGCAATCGGTC-3'). The resulting PCR product was digested with $\mathrm{NdeI}$ and $\mathrm{BamHI}$ (restriction sites are underlined in the primer sequences) and cloned into pSCrhaB2 (Cardona and Valvano, 2005; kindly provided by Prof. M. Valvano) that had been treated with the same restriction endonucleases.

The recombinant vector was then introduced into B. cenocepacia resistant mutants by conjugation via a triparental mating, as previously described (Craig et al., 
1989). The expression of BCAM1948 was induced by adding rhamnose $(0.2 \%)$ to the LB medium. The MIC of 10126109 , chloramphenicol, ciprofloxacin, levofloxacin, nalidixic acid, norfloxacin, and sparfloxacin were determined for both the mutant strains transformed with the empty vector and for the same strains carrying the BCAM1948/pSCrhaB2 vector, all grown in LB medium in the presence of trimethoprim $(800 \mu \mathrm{g} / \mathrm{ml})$ and $0.2 \%(\mathrm{w} / \mathrm{V})$ rhamnose.

\section{Results}

\section{Identification of a New Compound with Antimicrobial Activity Against B. cenocepacia}

More than 100 compounds from an in-house collection and shown to be effective against other bacteria were tested for their efficacy against B. cenocepacia J2315. For each compound, the MIC was evaluated through the twofold microdilution method (Martin et al., 2006); only a compound belonging to the 2,1,3benzothiadiazol-5-yl family (named 10126109, Figure 1), was found to have a promising antibacterial activity, showing a MIC of $8 \mu \mathrm{g} / \mathrm{ml}$ against B. cenocepacia J2315 (Table 1).

Cytotoxicity of the lead compound 10126109 was determined on HeLa cells and shown a 50\% cytotoxic concentration $\left(\mathrm{CC}_{50}\right)>100 \mu \mathrm{M}$. The compound was also tested for its

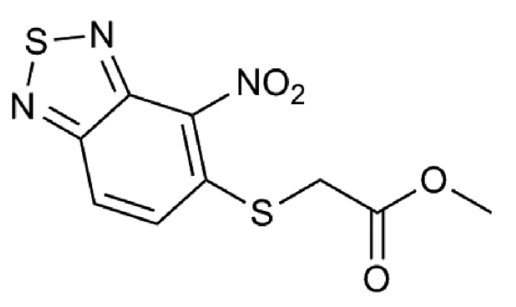

FIGURE 1 | Chemical structure of 10126109 compound.

TABLE 1 | Antimicrobial susceptibilities $(\mu \mathrm{g} / \mathrm{ml}$ ) of Burkholderia cenocepacia J2315, of 10126109 resistant mutants, and of the complemented mutants.

\begin{tabular}{llllllll}
\hline $\begin{array}{l}\text { B. cenocepacia } \\
\text { strains }\end{array}$ & \multicolumn{7}{c}{ Compound MIC $(\boldsymbol{\mu} \mathbf{g} / \mathbf{m l})$} \\
\cline { 2 - 8 } & $\mathbf{1 0 1 2 6 1 0 9}$ & $\mathbf{C H L}$ & $\mathbf{C I P}$ & $\mathbf{L V X}$ & $\mathbf{N A L}$ & NOR & SPX \\
\hline J2315 & 8 & 32 & 4 & 4 & 16 & 16 & 8 \\
SB5 & 128 & 64 & 32 & 32 & 256 & 128 & 64 \\
SB6 & 128 & 64 & 32 & 32 & 256 & 128 & 64 \\
SB34 & 256 & 64 & 32 & 32 & 256 & 128 & 64 \\
SB36 & 256 & 64 & 32 & 64 & 256 & 128 & 128 \\
SB5/pSCrhaB2 & 64 & 64 & 32 & 16 & 256 & 128 & 64 \\
SB5/pSCrhaB2-1948 & 16 & 32 & 16 & 16 & 32 & 32 & 16 \\
SB34/pSCrhaB2 & 64 & 64 & 32 & 16 & 256 & 128 & 64 \\
SB34/pSCrhaB2-1948 & 8 & 32 & 8 & 8 & 32 & 32 & 16 \\
SB36/pSCrhaB2 & 128 & 64 & 64 & 32 & 256 & 128 & 64 \\
SB36/pSCrhaB2-1948 & 8 & 32 & 8 & 8 & 32 & 32 & 16
\end{tabular}

CHL, chloramphenicol; CIP, ciprofloxacin; LVX, levofloxacin; NAL, nalidixic acid; NOR, norfloxacin; SPX, sparfloxacin. potential mutagenicity using the SOS-chromotest at $50 \mu \mathrm{M}$ and the test was negative. 10126109 has good pharmacokinetic properties, low toxicity and a scaffold already used in commercial compounds (e.g., Tizanidine hydrochloride).

In order to assess if 10126109 exerts a bacteriostatic or a bactericidal effect on Burkholderia cells, a time-kill curve was constructed using concentrations ranging from 0.5 to 4 times the MIC value (Figure 2). A bactericidal effect was observed using two and fourfold the MIC of 10126109 (i.e., a reduction of $99.9 \%$ $(\geq 3 \log 10)$ of the total number of CFU ml $\mathrm{m}^{-1}$ in the original inoculum was observed), while the number of $B$. cenocepacia cells remained constant when an amount of compound equal to the MIC was added to the culture. These data suggest that bacterial killing is concentration dependent, as already reported for ciprofloxacin (Silva et al., 2011).

To assess if 10126109 is active against clinical circulating strains, the compound was tested against $30 \mathrm{~B}$. cenocepacia clinical isolates (belonging to genomovars III-A, III-B, and IIID). All the strains resulted to be susceptible to 10126109 , with MIC values ranging from $<2$ to $32 \mu \mathrm{g} / \mathrm{ml}$, except three strains belonging to genomovar III-B, which showed MICs of 64$256 \mu \mathrm{g} / \mathrm{ml}$ (Supplementary Table S1). These data provide a good starting point for pre-clinical trials.

Subsequently, the compound was tested also against members of all the other Bcc species (Supplementary Table S2), showing MIC values ranging from $8 \mu \mathrm{g} / \mathrm{ml}$ (B. ambifaria, B. anthina, B. arboris, and B. lata) to $32 \mu \mathrm{g} / \mathrm{ml}$ (B. stabilis, B. ubonensis, and B. vietnamiensis).

\section{Isolation and Characterization of Spontaneous Mutants Resistant to 10126109}

To isolate spontaneous resistant mutants in an attempt to find the 10126109 target(s), B. cenocepacia J2315 cells were spread on plates containing 4-20-fold the MIC of the compound.

Two spontaneous mutants isolated through direct selection onto solid medium containing $64 \mu \mathrm{g} / \mathrm{ml}$ of the benzothiadiazol derivative (eightfold the MIC), and two additional mutants, isolated from plates containing LB plus $128 \mu \mathrm{g} / \mathrm{ml}$ of the compound (16-fold the MIC) were selected for further characterization. These mutant strains were named SB5, SB6, SB34, and SB36, respectively, and the resistance phenotype was confirmed for all the mutants (Table 1). The mutation frequency was about $4 \times 10^{-9}$.

The genomic DNA of mutant strains was then extracted and sequenced by Illumina Solexa method (IGA Technology Services, Udine) with the aim to understand which mutations were responsible for the resistance profile. The only gene resulting mutated in all the strains was BCAM1948. It encodes a 159 amino acid protein, which belongs to the MerR family of transcriptional regulators. As it is located immediately downstream from the BCAM1945-BCAM1947 operon, encoding the putative quinoxaline efflux system transporter, previously named RND-9 (Bazzini et al., 2011), we hypothesized that this protein controls the expression of the efflux system.

The mutation identified in SB5 strain was the substitution C58T causing arginine-20 to be replaced by a cysteine residue. In 


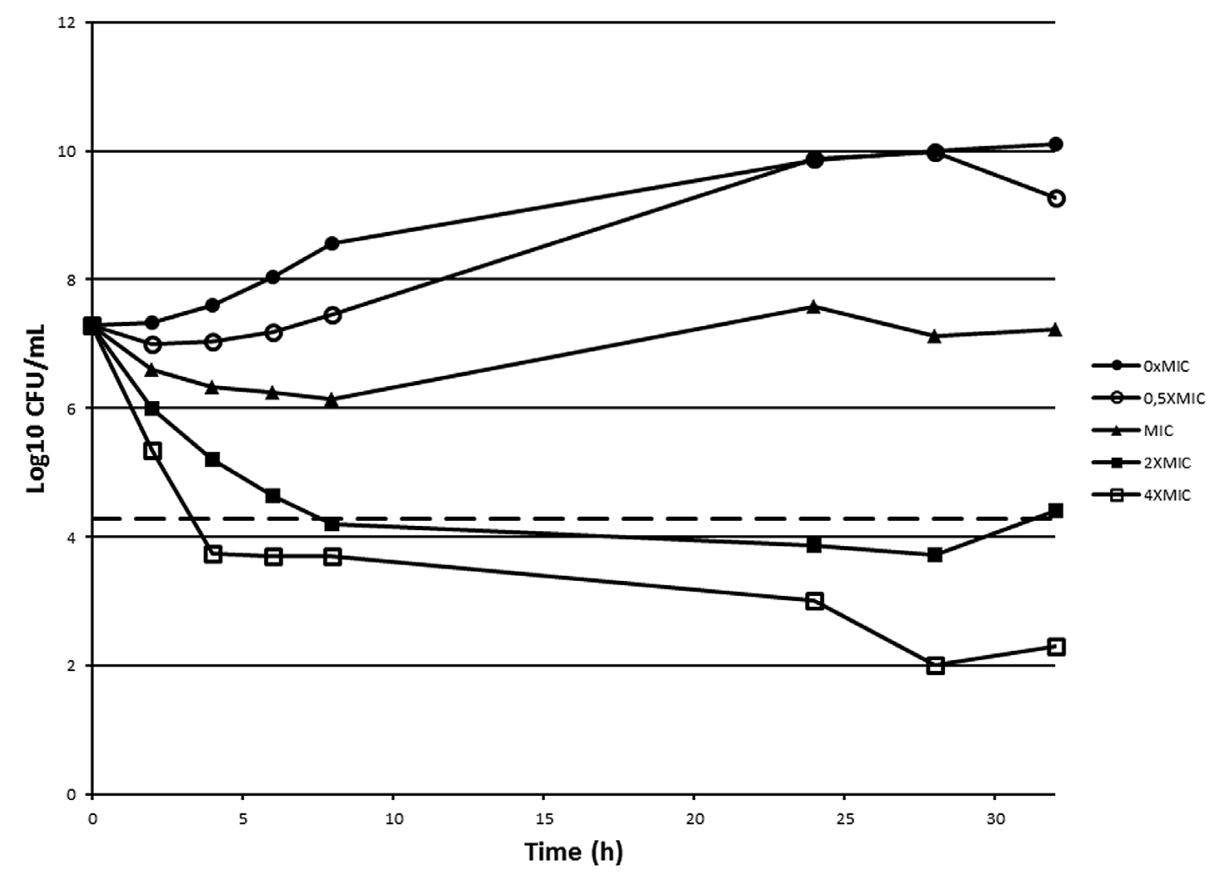

FIGURE 2 | Time-kill curve of Burkholderia cenocepacia J2315 exposed to 10126109. A B. cenocepacia J2315 culture in exponential phase of growth (•) was split and concentrations of 10126109 corresponding to $0.5(\bigcirc), 1(\mathbf{\Delta}), 2(\mathbf{\square})$, and 4-fold $(\square)$ the MIC value were added. The viable counts were determined at $37^{\circ} \mathrm{C}$ during $32 \mathrm{~h}$. The dotted line indicates a reduction of $3 \log 10$.

the SB36 resistant mutant, C-58 was changed into an A, leading to the replacement of arginine-20 with a histidine residue.

In SB6 mutant, the mutation was located in the intergenic region between BCAM1947 and BCAM1948, where A-49 is deleted. Interestingly, this deletion is located in a palindromic sequence $\left(5^{\prime}\right.$-ttgaagttaacttcaa- $\left.3^{\prime}\right)$.

In SB34 mutant, the mutation was the substitution T380C, which led to the amino acid change Leu-153 $\rightarrow$ Pro, having the two amino acids completely different chemical properties.

PCR amplification and sequencing were performed as described in Section "Materials and Methods" and confirmed the presence of the respective mutations in the genome of the four mutant strains.

Furthermore, we analyzed the evolutionary conservation and the possible functional consequences of the mutations falling within the coding region of BCAM1948. First, we checked the conservation of Arg-20 and Leu-153 in closely related microbes by aligning the best 30 BLAST hits (probing the NCBI non-redundant database) of the BCAM1948 encoded protein. A close inspection of this multialignment revealed that the Arg20 was conserved throughout all the analyzed genomes (see Supplementary Figure S1), suggesting the presence of some functional constraints acting in this position. Next, we checked the location of the mutated amino acids on the three-dimensional structure of the encoded protein. A search in Swiss Model Expasy (Biasini et al., 2014), revealed that the closest structural template of the MerR-like transcriptional regulator encoded by $B C A M 1948$ is the (2Fe-2S) oxidative-stress sensor SoxR from Escherichia coli (STML id: SMTL id: 2zhh.1, Watanabe et al.,
2008). The region (aa 1-80) embedding this residue (which resulted to be located at the accessible molecular surface) is predicted to be a DNA-binding domain. Interestingly, it has been shown that changes in the residues embedded in this region may lead to changes in the relative positions of all their protein subunits (5-helix, the DNA-binding domain and the Fe-S binding domain) and that the proper arrangement of the DNAbinding domain is essential for correct redox signal transduction (Watanabe et al., 2008). In particular, a change identical to the one identified in B. cenocepacia J2315 in this work (Arg-20 $\rightarrow$ Cys) has been already observed in E. coli (Hidalgo et al., 1997), where it leads to an altered redox phenotype. Accordingly, we here speculate that the most likely consequence of the changes in the Arg-20 position in B. cenocepacia J2315 mutants is an impaired redox activity of the BCAM1948 encoded protein.

Unfortunately, the other mutation in BCAM1948 (Leu$153 \rightarrow$ Pro) falls in a region showing no homology with other known 3D structures of MerR-like proteins and thus it is not possible to infer any feature of the altered phenotype resulting from this mutation in B. cenocepacia J2315.

\section{Expression Analysis of RND-9}

As the mutations responsible for the resistance to 10126109 were located in the gene encoding the transcriptional regulator BCAM1948, to determine whether this phenotype was associated with a differential expression of RND-9 system, quantitative RT-PCR experiments were performed. The expression levels of $B C A M 1946$, which codes for the RND transporter portion of RND-9, were assessed. Data obtained revealed that $r$ nd-9 gene 
was greatly and significantly up-regulated in all the mutants $(P<0.0001)$ in respect to the WT strain, the overexpression ranging between 200-fold in SB6 and about 1000-fold in SB36 (Table 2).

In order to confirm that the BCAM1946 overexpression established by qRT-PCR was related to the production of the corresponding protein, the membrane fractions of the B. cenocepacia WT and mutant strains were extracted and analyzed. The proteins present in this cellular fraction were analyzed by SDS-PAGE and Coomassie blue staining, as described in Supplementary data. A difference in the band pattern was observed between the WT and the four mutants, where a band of about $170 \mathrm{kDa}$ was more abundant in the mutants in respect to the J2315 strain (Supplementary Figure S2). This difference was confirmed by repeating the experiment with independent cultures. The band was excised from the acrylamide gel and identified by mass spectrometry. The band was identified as BCAM1946, the inner membrane component of RND-9 (Supplementary Table S3), which was shown to be overexpressed by qRT-PCR.

\section{Antibiotic Susceptibility of Mutant Strains and of the RND-9 Deleted Strain}

It is noteworthy that the overexpression of efflux pumps is responsible for a resistance phenotype in bacteria, being these transporters able to extrude different compounds. In this way, the MIC of different antibiotics was assessed for the four 10126109 resistant strains. The mutant strains showed a 216-fold increase in resistance toward all the compounds tested (Table 1). In particular, all of them were twofold more resistant to chloramphenicol, eightfold more resistant to ciprofloxacin, levofloxacin, norfloxacin, and sparfloxacin, and 16-fold more resistant to nalidixic acid compared to the WT strain (Table 1). SB36 mutant was 16-fold more resistant to levofloxacin and sparfloxacin (Table 1).

Although our previous finding suggested no correlation between RND-9 and ciprofloxacin resistance in B. cenocepacia (Buroni et al., 2014), being BCAM1948 a MerR transcriptional regulator, it could control the expression of other genes encoding both efflux pumps and/or involved in ciprofloxacin resistance.

To further assess whether RND-9 could be involved in 10126109 extrusion, the MIC of the compound was assessed also for the RND-9 deleted strain. In this case, the MIC was twofold lower respect to the WT.

Taken together these results confirmed that the RND-9 overexpression is responsible for resistance to 10126109, and

TABLE 2 | BCAM1946 expression levels by quantitative reverse
transcription PCR (qRT-PCR) in B. cenocepacia WT (J2315) and mutated
strains.
strains.

\begin{tabular}{ll}
\hline B. cenocepacia strains & BCAM1946 fold-change ( $(\mathbf{S D})$ \\
\hline J2315 & $1.16 \pm 0.85$ \\
SB5 & $855.97 \pm 12.58$ \\
SB6 & $205.07 \pm 16.30$ \\
SB34 & $469.22 \pm 81.30$ \\
SB36 & $993.66 \pm 72.99$
\end{tabular}

suggested the involvement of this transporter in the 10126109 efflux.

\section{Complementation}

To finally show that the mutations found in BCAM1948 gene were responsible for the resistant phenotype of the four B. cenocepacia mutants, SB5, SB34, and SB36 strains were transformed by triparental conjugation with pSCrhaB2 vector carrying a WT copy of BCAM1948. The expression of BCAM1948 was induced by adding $0.2 \%$ rhamnose to the growth medium (Cardona and Valvano, 2005).

Subsequently, the MIC of the compounds to which the four strains showed resistance, was assessed for the complemented strains (Table 1). The values were compared to the mutants transformed with the empty vector. Indeed, all the complemented strains showed MIC values (including those of 10126109) comparable to the B. cenocepacia J2315 strain (Table 1), indicating that complementation occurred.

Also these data confirmed the involvement of RND-9 in effluxmediated resistance to 10126109 .

These results, together with the overexpression of BCAM1946 shown by quantitative RT-PCR and membrane fraction extraction, indicate that BCAM1948 acts as a repressor for RND9. In fact, in the presence of a mutated regulator (strains SB5, SB34, and SB36) or of an altered putative operator region (strain SB6) the expression of the efflux pump was greatly increased. It is noteworthy that the mutations located in the repressor coding region lead to a greater overexpression of RND-9 compared to the mutation located in the operator (SB6).

The MerR regulators have been originally described as activators (Brown et al., 2003), even if a different role has been hypothesized and a double function previously reported (Lee et al., 1993). Our results strongly suggest that MerRlike regulators can act as repressors in $B$. cenocepacia, in agreement with a recent work revealing their role as repressors in Pseudomonas aeruginosa (Chambers and Sauer, 2013).

\section{Discussion}

It is noteworthy that unfortunately pharmaceutical industry is not investing in drug discovery against neglected infections such as those caused by $B$. cenocepacia, a dangerous CF pathogen. $\mathrm{CF}$ is a rare disease, and $B$. cenocepacia infections only occur in the $1-3 \%$ of patients. In this context, finding new molecules active against $B$. cenocepacia is of great importance as it is able to cause fatal infections which also lead the patient not to be admitted in transplant lists (Noone, 2008; De Soyza et al., 2010).

In this work, a compound belonging to the 2,1,3benzothiadiazol-5-yl family has been described as a promising bactericidal anti-Burkholderia agent, being the MIC against $B$. cenocepacia $\mathrm{J} 23158 \mu \mathrm{g} / \mathrm{ml}$. The compound has been shown to be effective also against $B$. cenocepacia clinical isolates and Bcc belonging species, thus providing a good starting point for pre-clinical trials. It is noteworthy that the compound is not cytotoxic, nor mutagenic, suggesting its potential safety 
for future clinical trials on humans. The chemical structure of this compound will be useful also for future research and structureactivity relationship studies.

Even if there is not a formal eradication protocol for Bcc infections, often combinations of two or three drugs are administered, including nebulised tobramycin (MIC $=256 \mu \mathrm{g} / \mathrm{ml}$ ) with or without additional oral antibiotics, such as minocycline ( $\mathrm{MIC}=16 \mu \mathrm{g} / \mathrm{ml}$ ) or meropenem (MIC $=64 \mu \mathrm{g} / \mathrm{ml}$; Horsley et al., 2011). EUCAST guidelines established that clinicians must continue to assess each patient individually and that it is not currently possible to establish MIC breakpoints for Bcc organisms ${ }^{3}$. In this context, the availability of a new chemical structure, showing an MIC lower respect to the currently used drugs, is a good and fundamental starting point to work on, in order to find new therapeutic solutions.

A mechanism of resistance to this new compound, which relies on the overexpression of RND-9 efflux pump, has been disclosed. Evidences came from the whole genome sequences of four resistant strains, showing mutations in the MerR transcriptional regulator BCAM1948 and responsible for the overexpression of the efflux pump both at the transcriptional and translational levels. Moreover, MIC determination of different compounds plus the complementation of the mutated strains with a WT copy of the gene confirmed the prominent role of the efflux pump in the resistance.

\footnotetext{
${ }^{3}$ http://www.eucast.org/fileadmin/src/media/PDFs/EUCAST_files/General_ documents/BCC_susceptibility_testing_130719.pdf
}

\section{References}

Aaron, S. D., Ferris, W., Henry, D. A., Speert, D. P., and Macdonald, N. E. (2000). Multiple combination bactericidal antibiotic testing for patients with cystic fibrosis infected with Burkholderia cepacia. Am. J. Respir. Crit. Care Med. 161, 1206-1212. doi: 10.1164/ajrccm.161.4.9907147

Bazzini, S., Udine, C., Sass, A., Pasca, M. R., Longo, F., Emiliani, G., et al. (2011). Deciphering the role of RND efflux transporters in Burkholderia cenocepacia. PLoS ONE 6:e18902. doi: 10.1371/journal.pone.0018902

Biasini, M., Bienert, S., Waterhouse, A., Arnold, K., Studer, G., Schmidt, T., et al. (2014). SWISS-MODEL: modelling protein tertiary and quaternary structure using evolutionary information. Nucleic Acids Res. 42, W252-W258. doi: 10.1093/nar/gku340

Brown, N. L., Stoyanov, J. V., Kidd, S. P., and Hobman, J. L. (2003). The MerR family of transcriptional regulators. FEMS Microbiol. Rev. 27, 145-163. doi: 10.1016/S0168-6445(03)00051-2

Buroni, S., Matthijs, N., Spadaro, F., Van Acker, H., Scoffone, V. C., Pasca, M. R., et al. (2014). Differential role of RND efflux pumps in antimicrobial drug resistance of sessile and planktonic Burkholderia cenocepacia cells. Antimicrob. Agents Chemother. 58, 7424-7429. doi: 10.1128/AAC.03800-14

Buroni, S., Pasca, M. R., Flannagan, R. S., Bazzini, S., Milano, A., Bertani, I., et al. (2009). Assessment of three Resistance-Nodulation-Cell Division drug efflux transporters of Burkholderia cenocepacia in intrinsic antibiotic resistance. BMC Microbiol. 9:200. doi: 10.1186/1471-2180-9-200

Cardona, S., and Valvano, M. A. (2005). An expression vector containing a rhamnose-inducible promoter provides tightly regulated gene expression in Burkholderia cenocepacia. Plasmid 54, 219-228. doi: 10.1016/j.plasmid.2005.03.004

Chambers, J. R., and Sauer, K. (2013). The MerR-like regulator BrlR impairs Pseudomonas aeruginosa biofilm tolerance to colistin by repressing PhoPQ. J. Bacteriol. 195, 4678-4688. doi: 10.1128/JB.00834-13
Our data support our previous studies on the role of B. cenocepacia efflux transporters in drug resistance (Buroni et al., 2009, 2014; Bazzini et al., 2011; Coenye et al., 2011; Scoffone et al., 2014). Among 16 efflux systems encoded in the genome of $B$. cenocepacia, only a few appear responsible for a resistant phenotype (RND-3, RND-4, RND-9, and RND-10). In this way, the use of new efflux inhibitors able to block these specific pumps coupled to new drugs able to interfere with $B$. cenocepacia growth, in order to avoid their extrusion, seems of primary importance in order to fight a main concern in Gram-negatives.

\section{Acknowledgments}

This work was supported by the Italian Cystic Fibrosis Research Foundation (FFC project \#10/2012 to G. R.; adopted by Associazione Trentina FC onlus in ricordo di Zaira Tutino, Gruppo di Sostegno FFC di Palermo - in ricordo di Elisa Pepe, Delegazione FFC di Imola).

The authors are grateful to Dr. P. Drevinek (University Hospital Motol, Charles University, Prague, Czech Republic) for providing some of the B. cenocepacia clinical isolates.

\section{Supplementary Material}

The Supplementary Material for this article can be found online at: http://journal.frontiersin.org/article/10.3389/fmicb. 2015.00815

Coenye, T., Van Acker, H., Peeters, E., Sass, A., Buroni, S., Riccardi, G., et al. (2011). Molecular mechanisms of chlorhexidine tolerance in Burkholderia cenocepacia biofilms. Antimicrob. Agents Chemother. 55, 1912-1919. doi: 10.1128/AAC.01571-10

Cox, M. P., Peterson, D. A., and Biggs, P. J. (2010). SolexaQA: at-a-glance quality assessment of Illumina second-generation sequencing data. BMC Bioinformatics 11:485. doi: 10.1186/1471-2105-11-485

Craig, F. F., Coote, J. G., Parton, R., Freer, J. H., and Gilmour, N. J. (1989). A plasmid which can be transferred between Escherichia coli and Pasteurella haemolytica by electroporation and conjugation. J. Gen. Microbiol. 135, 28852890. doi: 10.1099/00221287-135-11-2885

De Soyza, A., Meachery, G., Hester, K. L., Nicholson, A., Parry, G., Tocewicz, K., et al. (2010). Lung transplantation for patients with cystic fibrosis and Burkholderia cepacia complex infection: a single-center experience. J. Heart Lung Transplant. 9, 1395-1404. doi: 10.1016/j.healun.2010.06.007

Dodge, J. A., Lewis, P. A., Stanton, M., and Wilsher, J. (2007). Cystic fibrosis mortality and survival in the UK: 1947-2003. Eur. Respir. J. 29, 522-526. doi: 10.1183/09031936.00099506

Hidalgo, E., Ding, H., and Demple, B. (1997). Redox signal transduction: mutations shifting [2Fe-2S] centers of the SoxR sensor-regulator to the oxidized form. Cell 88, 121-129. doi: 10.1016/S0092-8674(00)81864-4

Higgins, C. F. (2007). Multiple molecular mechanisms for multidrug resistance transporters. Nature 446, 749-757. doi: 10.1038/nature05630

Horsley, A., Webb, K., Bright-Thomas, R., Govan, J., and Jones, A. (2011). Can early Burkholderia cepacia complex infection in cystic fibrosis be eradicated with antibiotic therapy? Front. Cell. Infect. Microbiol. 1:18. doi: 10.3389/fcimb.2011.00018

Koboldt, D. C., Chen, K., Wylie, T., Larson, D. E., McLellan, M. D., Mardis, E. R., et al. (2009). VarScan: variant detection in massively parallel sequencing of individual and pooled samples. Bioinformatics 25, 2283-2285. doi: 10.1093/bioinformatics/btp373 
Lee, I. W., Levrelli, V., Park, S.-J., Totis, P. A., and Summers, A. O. (1993). In vivo DNA-protein interactions at the divergent mercury resistance (mer) promoters. J. Biol. Chem. 268, 2632-2639.

Lee, W. P., Stromberg, M. P., Ward, A., Stewart, C., Garrison, E. P., and Marth, G. T. (2014). MOSAIK: a hash-based algorithm for accurate next-generation sequencing short-read mapping. PLOS ONE 9:e90581. doi: 10.1371/journal.pone.0090581

LiPuma, J. J. (2010). The changing microbial epidemiology in cystic fibrosis. Clin. Microbiol. 23, 299-323. doi: 10.1128/CMR.00068-09

Livak, K. J., and Schmittgen, T. D. (2001). Analysis of relative gene expression data using real-time quantitative PCR and the $2^{-\Delta \Delta C T}$ method. Methods 25 , 402-408. doi: 10.1006/meth.2001.1262

Martin, A., Takiff, H., Vandamme, P., Swings, J., Palomino, J. C., and Portaels, F. (2006). A new rapid and simple colorimetric method to detect pyrazinamide resistance in Mycobacterium tuberculosis using nicotinamide. J. Antimicrob. Chemother. 58, 327-331. doi: 10.1093/jac/dkl231

NCCLS. (1999). Methods for Determining Bactericidal Activity of Antimicrobial Agents: Approved Guideline M26-A. Wayne: National Committee for Clinical Laboratory Standards.

Noone, P. G. (2008). Lung transplant and cystic fibrosis: what's new from the UK and France? Thorax 63, 668-670. doi: 10.1136/thx.2008.099622

Perrin, E., Fondi, M., Papaleo, M. C., Maida, I., Emiliani, G., Buroni, S., et al. (2013). A census of RND superfamily proteins in the Burkholderia genus. Future Microbiol. 8, 923-937. doi: 10.2217/fmb.13.50

Quillardet, P., Huisman, O., D’Ari, R., and Hofnung, M. (1982). SOS chromotest, a direct assay of induction of an SOS function in Escherichia coli K-12 to measure genotoxicity. Proc. Natl. Acad. Sci. U.S.A. 79, 5971-5975. doi: 10.1073/pnas.79.19.5971

Saiman, L., Siegel, J., and Cystic Fibrosis Foundation. (2003). Infection control recommendations for patients with cystic fibrosis: microbiology, important pathogens, and infection control practices to prevent patient-to-patient transmission. Infect. Control. Hosp. Epidemiol. 24, S6-S52. doi: 10.1086/ 503485

Schmidtke, M., Schnittler, U., Jahn, B., Dahse, H., and Stelzner, A. (2001). A rapid assay for evaluation of antiviral activity against coxsackie virus B3, influenza virus A, and herpes simplex virus type 1. J. Virol. Methods 95, 133-143. doi: 10.1016/S0166-0934(01)00305-6

Scoffone, V. C., Spadaro, F., Udine, C., Makarov, V., Fondi, M., Fani, R., et al. (2014). Mechanism of resistance to an antitubercular 2-thiopyridine derivative that is also active against Burkholderia cenocepacia. Antimicrob. Agents Chemother. 58, 2415-2417. doi: 10.1128/AAC.02438-13

Silva, F., Lourenço, O., Queiroz, J. A., and Domingues, F. C. (2011). Bacteriostatic versus bactericidal activity of ciprofloxacin in Escherichia coli assessed by flow cytometry using a novel far-red dye. J. Antibiot. (Tokyo) 64, 321-325. doi: 10.1038/ja.2011.5

Watanabe, S., Kita, A., Kobayashi, K., and Miki, K. (2008). Crystal structure of the $[2 \mathrm{Fe}-2 \mathrm{~S}]$ oxidative-stress sensor SoxR bound to DNA. Proc. Natl. Acad. Sci. U.S.A. 105, 4121-4126. doi: 10.1073/pnas.0709188105

Conflict of Interest Statement: The authors declare that the research was conducted in the absence of any commercial or financial relationships that could be construed as a potential conflict of interest.

Copyright (c) 2015 Scoffone, Ryabova, Makarov, Iadarola, Fumagalli, Fondi, Fani, De Rossi, Riccardi and Buroni. This is an open-access article distributed under the terms of the Creative Commons Attribution License (CC BY). The use, distribution or reproduction in other forums is permitted, provided the original author(s) or licensor are credited and that the original publication in this journal is cited, in accordance with accepted academic practice. No use, distribution or reproduction is permitted which does not comply with these terms. 